\title{
Convalescent COVID-19 Plasma
}

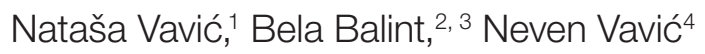

\begin{abstract}
Coronavirus disease 2019 (COVID-19), caused by severe acute respiratory syndrome coronavirus 2 (SARS-CoV-2), is a new human disease. December 31, 2019 marked the day the World Health Organization (WHO) first became aware of an infectious outbreak in the Hubei province in China. Until January 2021, more than two million people died from COVID-19. The use of convalescent plasma (CP) has been widely used in different outbreaks as the first therapeutic option given the lack of effective medications or vaccines, and often as a last chance or experimental treatment. CP is a strategy of passive immunisation. Possible mechanisms of CPCOVID-19 action are antiviral and immunomodulatory.

The established protocol for CP-COVID-19 collection defines activities and criteria related to recruiting and informing potential CP donors, clinical and laboratory examination, plasma collection, labelling and storage. Plasma is collected by apheresis/plasmapheresis. Administration of plasma is performed at the request of clinicians, according to the strict indications based on the severity of clinical picture, expressed by precisely determined "scoring" of symptoms. The risks transfusion recipients are likely to be exposed to do not differ from those of standard plasma recipients. At the Blood Transfusion Institute of Serbia, the first plasmapheresis from the recovered patient-donor was performed on 11 April 2020 and so far, collection and distribution of CP-COVID-19 have been performed continuously.

During the observation period, preliminary results of the effect of CP transfusion, along with other applied therapy, indicate its favourable effect, both worldwide and in Serbia. CP-COVID-19 should be used as early as possible in the course of infection in order to achieve the best outcomes.
\end{abstract}

Keywords: Coronavirus disease 2019 (COVID-19); Acute respiratory syndrome coronavirus 2 (SARS-CoV-2); Convalescent plasma.
(1) Blood Transfusion Institute of Serbia, Belgrade, Serbia.

(2) Institute of Cardiovascular Diseases "Dedinje", Belgrade, Serbia.

(3) Serbian Academy of Sciences and Arts, Belgrade, Serbia.

(4) Military Medical Academy, Belgrade, Serbia.
Correspondence:

NATAŠA VAVIĆ

E: najavavic@gmail.com

\section{ARTICLE INFO}

Received: 22 March 2021 Accepted: 22 March 2021

\section{Introduction}

Coronavirus disease 2019 (COVID-19) is a new disease caused by the severe acute respiratory syndrome coronavirus 2 (SARS-CoV-2). The disease is manifested in the human population in a wide range of clinical picture and severity, from almost asymptomatic and mild disease of the upper respiratory tract, to diffuse pneumonia accompanied by acute respiratory failure with a fatal outcome. In addition, it quickly became clear that the disease was characterised by a number of severe and unexpected complications, such as systemic thrombosis, cytokine storm syndrome, multiorgan dysfunction syndrome, as well as long-lasting and severe sequelae after infection, such as pulmonary fibrosis and major or minor damage to many other organs. ${ }^{1}$ Patients with COVID-19 infection show similar clinical signs and symptoms as patients with SARS-CoV and 
Middle East respiratory syndrome coronavirus (MERS-CoV) infection in terms of fever, dyspnoea and multilocular invasive changes on lung images, as well as the occurrence of multiorgan failure in severe forms of the diseases. ${ }^{2}$

December 31, 2019 marked the day when the World Health Organization (WHO) first became aware of the COVID-19 infection in the province of Hubei in China and already in January 2021 more than two million people had died from the effects of COVID-19 (https: //coronavirus.jhu.edu/map. html). ${ }^{3}$ Given the lack of natural immunity in the population to this virus, as well as the fact that there are currently no established effective therapeutic protocols for this disease and that vaccination of the population is practically just beginning, it can be concluded that, for the time being, the treatment of this infection remains mostly supportive.

Convalescent plasma (CP) has previously been widely used in various epidemics as the first therapeutic option in conditions where there were no effective drugs or vaccines and has often been used as the last resort or experimental treatment. ${ }^{4}$ This method, as a strategy of passive immunisation, has been used in the prevention and treatment of infectious diseases since the beginning of the $20^{\text {th }}$ century, although the principle of therapeutic infusion of CP was established in 1890, when it was used in the treatment of diphtheria. It should also be emphasized that the first Nobel Prize in Physiology and Medicine was awarded for the serum therapy of patients with diphtheria in 1901. This therapeutic method has recently been applied to other respiratory viral infections, including SARS-CoV-1, H1N1 influenza, and MERS-CoV, as well as West Nile virus and Ebola virus. ${ }^{5}$ In the current pandemic, it can also be said that one of the current therapeutic options for the treatment of COVID-19 infection is CP-anti-COVID-19 (CP-COVID-19) which is collected from people who had recovered from COVID-19 infection and which contains antibodies to SARS-CoV-2.6,

\section{Possible mechanisms of action of CP-COVID-19}

Possible mechanisms of action CP-COVID-19 is antiviral and immunomodulatory. The antiviral efficacy of CP treatment in COVID-19 infection is thought to be related to the activity of plasma neutralising antibodies (NAbs) in the blood of re- covered patients. These antibodies are crucial in neutralising and eliminating the virus and play a key role in protecting against viral infections. $\mathrm{CP}$ containing these antibodies contributes to the passive immunity and can help the body to fight infection more successfully. It is very important that these neutralising antibodies found in CP in patients infected with SARS-CoV-2 act antivirally by suppressing the replication of the virus even before the infected person begins to establish a humoral immune response to the infection.

Studies have shown that the NAbs titre in patients infected with SARS-CoV-2 was low when measured up to 10 days after the onset of the disease and then increased and that the highest antibody concentration was registered 10 to 15 days after the onset of the disease, after which the NAbs titre remained stable. ${ }^{8,9}$

The immunomodulatory effect of CP is based on the fact that the plasma of healthy donors contains anti-inflammatory cytokines and antibodies that block complement, pro-inflammatory cytokines and autoantibodies, all of which contribute to the immunomodulatory effect of $\mathrm{CP}$ in patients with COVID-19. Immunomodulatory effects of CP-COVID-19, in addition to immunoglobulins and other antibodies that inhibit the complement cascade and limit the formation of immune complexes, are also achieved by specific immunoglobulins $G$ that neutralise cytokines such as IL-1b, IL- 6 and TNF $\alpha$ that may play an important role in pathogenesis of uncontrolled degree of the inflammation which is seen in some patients with COVID-19 infection. ${ }^{10}$ In addition to the above, other proteins are obtained from donors, such as clotting factors, natural antibodies, defensin, pentraxin and other undefined proteins that may be important in the body's defences. Thus, the passive immunity obtained by infusion of CP-COVID-19 can limit the excessive and unfavourable inflammatory cascade triggered by pathogenic antibodies, as well as cell damage caused by the activation of the complement cascade due to the excessive inflammatory response. ${ }^{4}$ It should be emphasized, once again, that the described effect of immunomodulation can be very important, given that excessive activation of the immune system with systemic hyper-inflammation or the "cytokine storm" triggered by IL1b, IL-2, IL-6, IL-17, IL -8, TNF $\alpha$ in some patients with COVID-19 may be responsible for multiorgan dysfunction and death, as well as chronic damage 
to the lung parenchyma with consequent fibrosis and decreased lung function after recovery from COVID-19 infection. ${ }^{11}$

In some severely ill patients, it has been shown that the favourable immunomodulatory effect of CP-COVID-19 could be a consequence of the proven presence of $\operatorname{IgA}$ antibodies against cardiolipin and IgA and IgG antibodies to $\beta 2$-glycoprotein I. $^{12}$ By neutralising these proteins, CPCOVID-19 can reduce the risk of thrombo-embolic complications (ie, diseases similar to antiphospholipid syndrome), which are not uncommon in critically ill patients. Moreover, other antibody-mediated immune response mechanisms, such as antibody-induced complement activation and antibody-induced cellular cytotoxicity and phagocytosis, may also be the reason for the beneficial therapeutic effect of CP. Meffre and Ivasa$\mathrm{ki}^{13}$ showed that the defective genome for IFN-1, which is a genetically induced mutation on the $\mathrm{X}$ chromosome, is the cause of IFN-1 deficiency resulting in an uncontrolled replication and spread of the virus. INF-1 deficiency results in the suppression of immune-signalling complexes called inflammasomes and the increased production of inflammatory cytokines that are produced without the control of these complexes. These facts may explain the very serious course of COVID-19 infection in people with IFN-1 deficiency, as well as the benefits of using covalent plasma in these patients. $^{13}$

\section{Collection of CP-COVID-19}

The CP-COVID-19 collection protocol defines activities and criteria related to the recruitment and information of potential CP donors, clinical and laboratory examination, as well as plasma collection, labelling and storage. These criteria are in line with current versions of the Recommendations of the Council of Europe and the European Committee, the Food and Drug Administration (FDA), the World Health Organization (WHO). ${ }^{14-16}$

CP donors must be eligible for CP donation. They should have undergone COVID-19 infection, whether confirmed by an approved molecular test (eg, nasopharyngeal swab) or by the presence of antibodies to SARS-CoV-2. It is necessary that at least 14 days have passed after the cessation of clinical symptoms of the infection. In addition to this, they must also meet the criteria that apply to regular apheresis procedures. Potential $\mathrm{CP}$ donors undergo the same procedure as regu- lar voluntary blood donors. They fill in a standard questionnaire for blood donors and are subject to the usual medical physical examination. They perform laboratory tests as well as for regular plasma donors, including antibodies against SARS-CoV-2. According to FDA guidelines, a sufficient titre of neutralising SARS-CoV-2 antibodies for plasma donation is considered to be greater than or equal to $1: 160 .{ }^{17}$

Plasma collection is performed by apheresis procedure - plasmapheresis. This procedure involves effusing the donor's blood, centrifuging the blood, isolating and retaining a certain volume of plasma, usually about $600 \mathrm{~mL}$, and reinfusing the cellular components. Most often, the obtained amount of plasma is divided into 2-3 units that can be transfused separately. The procedure is performed on special devices, separators of blood components. Sets that are sterile, disposable, with the use of anticoagulants and preservatives are used. The procedure lasts about 40-60 min. The usual time period between the two procedures is 15 days, but it can be shorter depending on the concentration of IgG immunoglobulin, but not shorter than 4 days. Plasma units are frozen within $24 \mathrm{~h}$ of collection and are issued only after obtaining the results of a standard test of plasma donors. The procedure for storing $\mathrm{CP}$ is the same as for fresh frozen plasma - up to 36 months, at a temperature lower than $-25{ }^{\circ} \mathrm{C} .{ }^{14}$ COVID-19 CP must be appropriately labelled. In addition to the usual label for fresh frozen plasma, there must be a label that it is in experimental use or for clinical trials. Plasma recipient blood group and plasma unit must be compatible in the ABO blood group system.

CP-COVID-19 is issued at the request of the doctor treating the patient. Plasma administration is performed according to standard procedure and strict indications. The usual optimal dose is two units CP-COVID-19 or 4-13 mL/kg body weight of the recipient. ${ }^{18,19}$

\section{Safety and efficiency of application CP- COVID-19}

In March 2020, the FDA published a guidance document regarding CP-COVID-19. ${ }^{16}$ On $23 \mathrm{Au}-$ gust 2020 the FDA issued an Emergency Use Authorization (EUA) for CP-COVID-19 for the treatment of hospitalised patients with COVID-19.20 The FDA claimed that "overall evidence" suggest- 
ed that the benefits of $\mathrm{CP}$ would outweigh the risks, and given the lack of effective treatments, the FDA provided guidance on the production and use of CP in hospitalised patients with signs of progressive infection. The American Society of Infectious Diseases and the AABB (American Blood Bank Association) recommend that the use of CP should be limited to clinical trials. They believe that critically ill patients and those in the intensive care unit (ICU) are unlikely to benefit from CP transfusions, but that CP should still be used as early as possible during infection (preferably within 3 days of diagnosis) to achieve the best results. They believe that transfusion may be useful if high-titre anti-spike protein receptor binding domain in CP (RBD) IgG titre $\geq 1: 1350$ is administered early on admission to the hospital, within $72 \mathrm{~h}$, and that mortality is then reduced within 28 days after transfusion. ${ }^{21}$ Libster et al concluded that administration of a high titre of CP-COVID-19 to infected elderly within $72 \mathrm{~h}$ after the onset of mild symptoms reduced the progression of COVID-19 to severe disease. This simple and inexpensive intervention can save lives. ${ }^{22}$

The risk of a CP transfusion to recipients is no different from the risk posed by standard plasma transfusion. The risk of transfusion-transmitted infection is very low due to the performed tests. Non-infectious dangers of transfusion, such as allergic reactions, transfusion-related circulatory overload and transfusion-related acute lung injury (TRALI) are possible, but rare. However, routine donor screening includes donor human leukocyte antigen (HLA) antibodies with a history of pregnancy or previous transfusion to reduce the risk of TRALI. There are no reports of transmission of the respiratory virus by blood transfusion. The risk of transmission of SARS$\mathrm{CoV}-2$ by plasma transfusion is considered to be negligible. At the Mayo Clinic, from 11 July 2020 more than 34,000 patients were transfused with CP-COVID-19 (https://www.uscovidplasma.org) with minimal side effects. ${ }^{23}$ The first plasmapheresis of the first recovered patient-donor was performed at the Blood Transfusion Institute of Serbia on 11 April 2020 and, so far, the collection and distribution of CP-COVID-19 has been performed continuously. Finally, from our country there are also published data on antibody investigation and collection of convalescent plasma by plasmapheresis for basic research and/or potential therapeutic use. ${ }^{24}$

\section{Conclusion}

$\mathrm{CP}$ is a safe and potentially effective strategy for treating new pathogens, especially in those scenarios without proven antiviral agents, vaccines or specific anti-COVID-19 intravenous immunoglobulin (IVIg). Early infusions of CP can provide a bridge for recovery to the risky patients until vaccines become widely available. As IVIg and CP share similar mechanisms of action, the production of anti-COVID-19 IVIg may provide better standardisation in COVID-19 therapy. In this observed period, preliminary results of the effect of $\mathrm{CP}$ transfusion, together with other applied therapy, indicate its favourable effect, both in Serbia and worldwide. CPCOVID-19 should be used as early as possible during infection (preferably within 3 days of diagnosis) to achieve the best treatment results.

\section{Acknowledgements}

None.

\section{Conflict of interest}

None.

\section{References}

1. Richardson S, Hirsch JS, Narasimhan M, Crawford JM, McGinn T, Davidson KW, et al. Presenting characteristics, comorbidities and outcomes among 5700 patients hospitalized with COVID-19 in the New York City area. JAMA 2020 May 26;323(20):2052-59.

2. Huang C, Wang Y, Li X, Ren L, Zhao J, Hu Y, et al. Clinical features of patients infected with 2019 novel coronavirus in Wuhan, China. Lancet $2020 \mathrm{Feb}$ 15;395(10223):497-506.

3. Dong E, Du H, Gardner L. An interactive web-based dashboard to track COVID-19 in real time. Lancet Infect Dis 2020 May;20(5):533-4.

4. Garraud O, Heshmati F, Pozzetto B, Lefrere F, Girot $\mathrm{R}$, Saillol A, et al. Plasma therapy against infectious pathogens, as of yesterday, today and tomorrow. Transfus Clin Biol 2016 Feb;23(1):39-44. 
5. Marano G, Vaglio S, Pupella S, Facco G, Catalano L, Liumbruno GM, et al. Convalescent plasma: new evidence for an old therapeutic tool? Blood Transfus 2016 Mar;14(2):152-7.

6. Roback JD, Guarner J. Convalescent plasma to treat COVID-19: possibilities and challenges. JAMA $2020 \mathrm{Apr}$ 28;323(16):1561-2.

7. Chen L, Xiong J, Bao L, Shi Y. Convalescent plasma as a potential therapy for COVID-19. Lancet Infect Dis 2020 Apr;20(4):398-400.

8. Liu STH, Lin HM, Baine I, Wajnberg A, Gumprecht JP, Rahman F, et al. Convalescent plasma treatment of severe COVID-19: a propensity score-matched control study. Nat Med 2020 Nov;26(11):1708-13.

9. Wu F, Wang A, Liu M, Wang Q, Chen J, Xia S, et al. Neutralizing antibody responses to SARS-CoV-2 in a COVID-19 recovered patient cohort and their implications. medRxiv 2020.03.30.20047365; doi: https://doi. org/10.1101/2020.03.30.20047365.

10. Abe Y, Horiuchi A, Miyake M, Kimura S. Anti-cytokine nature of natural human immunoglobulin: one possible mechanism of the clinical effect of intravenous immunoglobulin therapy. Immunol Rev 1994 Jun;139:5-19.

11. McGonagle D, Sharif K, O'Regan A, Bridgewood C. The role of cytokines including interleukin- 6 in COVID-19 induced pneumonia and macrophage activation syndrome-like disease. Autoimmun Rev 2020;19(6):102537 doi: 10.1016/j.autrev.2020.102537.

12. Zhang $Y$, Xiao $M$, Zhang $S$, Xia $P$, Cao W, Jiang W, et al. Coagulopathy and antiphospholipid antibodies in patients with Covid-19. N Engl J Med 2020 Apr 23;382(17):e38. doi: 10.1056/NEJMc2007575.

13. Meffre E, Iwasaki A. Interferon deficiency can lead to severe COVID. Nature 2020 Nov;587(7834):374-6.

14. European Committee on Blood Transfusion. Guide to the preparation, use and quality assurance of blood components. 20th ed. Strasbourg, France: European Directorate for the Quality of Medicines and HealthCare, 2020.

15. U.S. Food and Drug Administration. Covid-19 Convalescent Plasma. EUA decision memo [Internet] [Updated 2020-Aug-23]. USA. [Cited: 2021-Mar-20]. Available from: https://www.fda.gov/media/141480/download.

16. WHO. WHO Guidance on maintaining a safe and adequate blood supply during the coronavirus disease
2019 (COVID-19) pandemic and on the collection of COVID-19 convalescent plasma. Interim guidance. [Internet] [Updated 2020-Jul-10]. Geneva. [Cited: 2021Mar-20]. Available from: https://apps.who.int/iris/ handle/10665/333182.

17. U.S. Food and Drug Administration. Center for Biologics Evaluation and Research. Revised Information for Investigational COVID-19 Convalescent Plasma. [Internet] [Updated 2021-Feb-12]. USA. [Cited: 2021-Mar-20]. Available from: https://www. fda.gov/vaccines-blood-biologics/investigational-new-drug-ind-or-device-exemption-ide-process-cber/recommendations-investigational-covid-19-convalescent-plasma.

18. Bloch EM, Shoham S, Casadevall A, Sachais BS, Shaz B, Winters JL, et al. Deployment of convalescent plasma for the prevention and treatment of COVID-19. J Clin Invest 2020 Jun 1;130(6):2757-65.

19. Li L, Zhang W, Hu Y, Tong X, Zheng S, Yang J, et al. Effect of convalescent plasma therapy on time to clinical improvement in patients with severe and life-threatening COVID-19: a randomized clinical trial. JAMA 2020 Aug 4;324(5):460-70

20. Tanne JH. Covid-19: FDA approves use of convalescent plasma to treat critically ill patients. BMJ 2020 Mar 26;368:m1256. doi: 10.1136/bmj.m1256.

21. Salazar E, Christensen PA, Graviss EA, Nguyen DT, Castillo B, Chen J, et al. Treatment of Coronavirus disease 2019 patients with convalescent plasma reveals a signal of significantly decreased mortality. Am J Pathol 2020 Nov;190(11):2290-303.

22. Libster R, Pérez Marc G, Wappner D, Coviello S, Bianchi A, Braem V, et al. Early high-titer plasma therapy to prevent severe Covid-19 in older adults. N Engl J Med 2021 Feb 18;384(7):610-8.

23. Joyner MJ, Wright RS, Fairweather D, Senefeld JW, Bruno KA, Klassen SA, et al. Early safety indicators of COVID-19 convalescent plasma in 5000 patients. J Clin Invest 2020 Sep 1;130(9):4791-7.

24. Balint B, Todorovic M, Andric Z, Jovicic M, Blagojevic G, Colic M. Long-term antibody-response monitoring following primary exposure to SARS-CoV-2 and afterward mRNA COVID-19 vaccination: a case report. Vojnosanit Pregl 2021. DOI: https://doi.org/10.2298/ VSP200220019B. 